\title{
An der Hand der Philosophen durch die Weltgeschichte
}

\section{Jürg Kesselring}

Prof. Dr. med., Chefarzt für Neurologie und Rehabilitation am Rehabilitationszentrum Valens

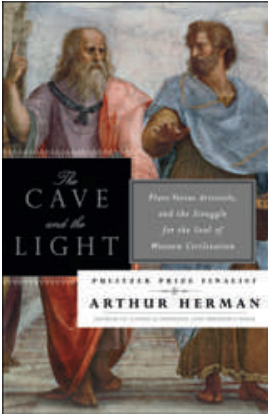

Arthur Herman

The Cave and

the Light

Plato Versus Aristotle, and the Struggle for the Soul of Western Civilization

New York: Penguin Random House; 2014 704 Seiten. 18 USD. ISBN 978-0-5533-8566-3
Lohnt es sich in Zeiten digitaler Erschöpfung, fortgeschrittenen Missvergnügens und verbreiteter gedanklicher Wurstigkeit überhaupt noch, dicke Bücher über tote weisse Männer zu lesen, die vor zweieinhalbtausend Jahren Griechisch schrieben und die Spuren deren Philosophierens durch die ganze Geschichte der westlichen Kulturen hindurch verfolgen? Meine Antwort nach der Lektüre dieses Buches von Arthur Hermann ist ein klares, freudiges und überzeugtes: Ja! Zumal, da ich als Neurologe diese beiden grossen Philosophen Platon und Aristoteles auch als Metaphern verwenden kann für das, was uns in den modernen Neurowissenschaften besonders beschäftigt: wie die beiden Hirn-Hemisphären unterschiedliche Funktionen und Aufgaben annehmen bzw. ausüben und wie wir, nicht nur als behandelnde Ärzte, sondern wir alle ein Gleichgewicht zwischen diesen beiden verschiedenen Arten des In-der-Welt-Seins und In-ihrHandelns anstreben und übend pflegen sollen.

Platon, der im Siebten Buch der Politeia das Höhlengleichnis beschreibt, in dem sich die Menschen als bestimmt von einer Schöpfung oder einem Schöpfer finden, die den Weg aus der dunklen Höhle der materiellen Existenz zum Lichte höherer, reinerer und spirituellerer Wahrheiten des Ewigen, Unsterblichen und Unveränderbaren finden sollten. Er ist selbst etwas enttäuscht, dass nur so wenige dieses Ziel anstreben und sich mit einem Dasein in diesem Dunkel und mit dem Anblick von Täuschungen zufrieden geben. In diesem Bild findet sich hinter den Menschen eine Mauer, auf der die Wirklichkeiten sich bewegen, von hinten angeleuchtet, sodass die Menschen immer nur deren Schatten auf der Wand vor ihnen sehen können, nicht aber die wahren Wirklichkeiten der einzig sinnhaften Ideen und Formen. Seine Schule ist die Akademie. Aristoteles aber, der Arztsohn, findet ganz andere Weisen, in der Welt zu sein und in ihr zu handeln und zu wirken. Er geht von den Fakten aus, will sie ergründen, klassifizieren, ordnen. Es gibt hier ein Wissen aus dem Experiment, er traut den Sinnen, sucht Systeme, indem er beobachtet, Kausalitäten findet in der Materie, in der Wirkung, in der Form und im Zweck. Theorie und Praxis gehören bei ihm zum Handwerk, das es zu erlernen und immer weiter zu verfeinern gilt. In der Ethik geht es ihm nicht um ein Wissen, was Ethik letztlich sei, sondern darum, in der Gemeinschaft ethisch zu handeln. Das Mass ist sein Massstab, die Besonnenheit eine besondere Tugend. Seine Schule ist das Lyceum, das er den neun Musen widmet und das später über die Bibliothek von Alexandria in die ganze Welt hinaus wirkt.

Beide Philosophen beschreiben Erkenntniswege, die voneinander ganz unterschiedlich sind, sich aber idealerweise ergänzen können. Sie sind je ähnlich der Art und Weise, wie wir die Funktionen der beiden Hirnhemisphären beschreiben. Für das Aristotelische: Aufgaben der linken Hirnhälfte, etwa: verbal, sequentiell, zeitlich, digital, logisch, analytisch, rational, westliches Denken ... für das Platonische: Funktionen der rechten Hirnhälfte: nicht-verbal, visuell-räumlich, gleichzeitig, räumlich, analog, ganzheitlich, synthetisch, intuitiv, östliches Denken ... [1]. Oder wie der Psychologe William James [2] in seinem Pragmatismus die Dualität beschreibt: tender-minded sind eher rationalistisch (going by principles), intellektualistisch, idealistisch, optimistisch, religiös, mit freiem Willen, monistisch, dogmatisch (man hört Platon!), während die tough-minded eher empirisch (going by facts), sinnlich, materialis-

\section{Diese beiden grossen Philosophen Platon und} Aristoteles können auch als Metaphern für die beiden Hirn-Hemisphären gesehen werden.

tisch, pessimistisch, nicht religiös, fatalistisch, pluralistisch, skeptisch sind (es klingt wie bei Aristoteles!).

Und nun führt uns der Autor anhand dieser sorgfältig beschriebenen Polaritäten durch die Geschichte der westlichen Welt mit Fragen, ob man selber ein guter Bürger sein könnte oder einen Philosophen als Führer brauche, geht mit Alexander dem Grossen, der ja von Aristoteles gelernt hat, auf seine Wege, hält dann wieder bei Plato inne, der Sparta bewundert hat und wird in den Neu-Plato eines Plotin eingeführt, für den alles Körperliche ein Gefängnis war. Dann wieder der Gegensatz, sozusagen das Umschwingen des Pendels bei Origenes, der das Körperliche als einen Übungsplatz 
verstand und sich sogar fragte, ob vielleicht die Seele, die von weit her komme, sich diesen Übungsplatz schon etwa vorgeburtlich ausgewählt haben könnte. Die Philosophen mussten sich ja mit dieser ganz neuen Dimension des explizit Nicht-Philosophischen, des rechthaberischen Behauptens, die wie eine Welle aus dem Nahen Osten zunächst friedlich, dann aber ausserordentlich gewaltsam in den Westen nach Europa drang, auseinandersetzen. Die Wende erfolgte unter Konstantin, der in der Nacht vor der legendären Schlacht an der Milvischen Brücke die Erscheinung gehabt hatte, dass er "in diesem Zeichen siegen" werde. Dadurch wurde dann die Vergesellschaftung der spirituellen Dimension mit den Machthabern und ihren -kämpfen unausweichlich. Vielleicht war ja der Heilige Augustinus noch "der letzte Christ», aber dann kamen auch in den Kirchen die Sucher und Infrage-Steller auf den Plan, Petrus Abaelard, Boëthius mit ihren (aristotelischen) Logiken, Zweifeln und Fragen, später (über arabische Einflüsse) auch Thomas Aquinas, William of Ockham mit seinem Rasiermesser und seinen Auseinandersetzungen mit dem Päpstlichen. Um 1400 schloss die Autorität des Aristoteles jedes Argument mit einer trockenen Virtuosität ohne Freude oder Gnade.

Mir scheint, die beiden Strömungen seien in der Renaissance in Florenz am besten im Gleichgewicht gewesen: Kunst hatte ebenso ihren geschätzten Platz und ihre wertvolle Bedeutung wie auch die Wissenschaft. Am Hofe der Medicis kamen Künstler und Wissenschaftler ins Gespräch, Masilio Ficino gründete eine neue Platonische Akademie in Florenz und übersetzte Platons Dialoge. Raffael schuf in den Stanzen der Segnatura mit der «Schule von Athen» ein Gemälde, auf dem Platon (mit den Gesichtszügen Leonardo da Vincis), den Timaios in der einen Hand und mit der anderen zum Himmel weisend mit dem jugendlichen Aristoteles im Gespräch ist, der seine Ethik mit sich trägt und auf die Erde verweist. Um diese beiden wohlgeordnet herumgruppiert sind die Vertreter philosophischer Schulen bis zu jener Zeit, mit knappen Andeutungen tiefgründig analysiert und lebensvoll dargestellt. Aber die Geschichte geht weiter: In der Reformation war Martin Luther überzeugt, dass es gleich der Satan selber gewesen sei, der dem Aristoteles seine Ethik diktiert habe, weil ja darin kein Platz mehr für Gnade sei. Er hatte ja sein Heil - und damit gleich auch dasjenige aller Leute - auf die vier Soli reduziert: solus Christus, sola fide, sola gratia, sola scriptura.

Es ging dann weiter über Galileo und die neue Wissenschaft, an dem mir besonders gefällt, wenn er sagt: «diejenigen, welche sich nur auf Autoritäten berufen, um Sicherheit zu gewinnen, ohne selbst nach Argu- menten zu suchen, um dies zu unterstützen, handeln absurd. [...] Sie schliessen die Augen vor dem Licht der Wahrheit und ich weiss nicht, ob ich darüber lachen oder weinen soll.» Galileo brauchte Gott nicht zu sehen, um an ihn zu glauben - er brauchte nur die Grösse seiner Schöpfung zu sehen und mit Staunen auf sie zu blicken. Was er nicht ertrug, waren arrogante Ignoranten. Bekannt ist ja, wie er von der Kirche zum Rückruf seiner Theorien gezwungen worden sei. Im Prozess, der ihm im April 1633 gemacht wurde, ging es allerdings mit keinem Wort um seine wissenschaftlichen Theorien, sondern nur darum, dass er einem päpstlichen Erlass früherer Jahre nicht nachgefolgt sei. Dies ist umso tragischer, als der damals amtierende Papst Urban VIII früher als Kardinal Maffeo Barberini ein enger Freund Galileis gewesen war ...

\section{Der Autor führt uns anhand dieser}

sorgfältig beschriebenen Polaritäten durch die Geschichte der westlichen Welt.

Durch die ganze weitere Geschichte lässt sich die Dichotomie verfolgen bei den Platonisten in Cambridge, zu denen Isaac Newton gehörte, dann aber wieder besonders die aristotelische Seite in der Zeit der Aufklärung mit wesentlichen Implikationen auf das politische Leben, dann wieder Rousseau als Platonist, der gleich alle Fakten wegrationiert haben wollte. Der Gegenpol zur Aufklärung in der Romantik mit einem Glauben an eine ewige und wohltätige Natur und die Präsenz transzendentaler moralisch Gesetze im Leben. Vielleicht war dort ein gewisser Ausgleich oder eine Übereinstimmung und Synthese von Aristoteles' Instinkt, die Vernunft in der materiellen Welt zu verankern und Platons Wunsch, unsere spirituelle innere Natur zu realisieren, vorübergehend möglich geworden. Und dann kam Hegel mit seinem Rückgriff auf Platon, der einen derart tiefen Einfluss auf die weiteren geschichtlichen Entwicklungen hatte, obwohl (oder gerade: weil?) er eine Sphinx war.

Auf der allerletzten Seite dieses ausführlichen, ausserordentlich kenntnisreich und spannend geschrieben Buches, das uns an Hand der beiden Athener Philosophen durch die Geschichte der Westlichen Welt in den letzten zweitausend Jahren führt, findet sich eine einzige Fussnote, die auf das wichtige Buch von Iain McGilchrist hinweist: The Master \& His Emissary. The Divided Brain and the Making of the Western World [3], das hier schon besprochen [4] und in eigener Weise ausgestaltet worden ist [5]. In guter Gesellschaft befinden wir uns, wenn Albert Einstein schreibt: «Der intuitive Geist ist ein heilige Gabe ... der rationale Geist ist ein treuer Diener ... und wir haben eine Gesellschaft kreiert, die den Diener ehrt, aber die Gabe vergessen hat.» 